ANNALES

POLONICI MATHEMATICI

$87(2005)$

\title{
Volume and multiplicities of real analytic sets
}

\author{
by Guillaume Valette (Kraków)
}

\begin{abstract}
We give criteria of finite determinacy for the volume and multiplicities. Given an analytic set described by $\{v=0\}$, we prove that the log-analytic expansion of the volume of the intersection of the set and a "little ball" is determined by that of the set defined by the Taylor expansion of $v$ up to a certain order if the mapping $v$ has an isolated singularity at the origin. We also compare the cardinalities of finite fibers of projections restricted to such a set.
\end{abstract}

1. Introduction. The question of sufficiency of jets has been studied by many authors [2], [3], [9], [13]. It allows one to compare a given singularity to a semi-algebraic one, which is very useful if one wants to do some effective computations.

In this paper we study the volume and multiplicities of real analytic germs. By volume we mean the Hausdorff measure. In [5] (see also [1]) it is proved that the volume of the intersection of a subanalytic set and a ball of radius $r$ has a Puiseux extension in $r$ and $\ln r$. We give here some explicit criteria for the determinacy of this expansion up to a certain order. The criterion we give is based on the Kuiper-Kuo criterion. By the Łojasiewicz inequality we get results of finite determinacy for mappings having an isolated singularity at the origin.

We also study the finite determinacy of multiplicities, that is, cardinalities of finite fibers of a projection restricted to a given analytic set. These numbers are important since they allow describing the topological type of a subanalytic set via cylindrical decompositions. Many theorems have been proved to enable effective computations of these multiplicities in the semialgebraic case. Therefore it is useful to have theorems which compare the multiplicities of a real analytic set to those of the set defined by the Taylor expansion of its equation.

2000 Mathematics Subject Classification: 32B20, 57N80, 58A20.

Key words and phrases: analytic sets, Hausdorff measure, stratification, sufficiency of jets.

This paper is partially supported by the RAAG Network. 
At the beginning we recall some results of D. Trotman and L. Wilson [9] about sufficiency of jets. They have proved that some particular transformation of "pull-back" type can improve the regularity of a stratification. In [11] the author proved that the volume is preserved under some topological trivializations. Then we prove our determination theorems for the volume and multiplicities.

\section{Notation and some definitions}

2.1. $\left(t^{i}\right)$ regular stratifications. The $\left(t^{i}\right)$ conditions have been introduced by $\mathrm{D}$. Trotman. They generalize a condition introduced by R. Thom which concerned $C^{\infty}$ manifolds. We recall the definition of [9]. Let

$$
\mathbb{G}^{d}=\bigcup_{j=m}^{d} \mathbb{G}(d ; j)
$$

where $\mathbb{G}(d ; j)$ is the Grassmannian of $j$-dimensional vector spaces in $\mathbb{R}^{d}$.

In what follows, $m$ and $n$ will denote fixed integers. We set $\mathbb{R}^{d}=\mathbb{R}^{n} \times \mathbb{R}^{m}$. We will denote by $N$ the subset $\mathbb{R}^{n}$ and by $Y$ the subset $\mathbb{R}^{m}$. The mapping $\pi$ will be the projection on $Y$ and $\pi^{\perp}$ will be the canonical projection on $N$.

We will denote by $X$ a subset of $\mathbb{R}^{d}$ and by $\mathcal{S}=\left\{Y, X_{1}, \ldots, X_{p}\right\}$ a stratification of $X$ by $C^{\infty}$ manifolds.

Definition 2.1.1. A direct transversal to $Y$ is the germ of an analytic mapping $v: N \rightarrow Y$. Its graph will be denoted by $\Gamma_{v}$.

Abusively we will say "transversal" instead of "direct transversal", since all the transversals in this paper will be direct ones.

First we recall the definition of $\left(t^{i, j}\right)$ conditions. As in [9] we will denote by $\mathbb{R}^{-}$the set of real numbers marked with the symbol - in exponent. For $i \in \mathbb{R}$ we will say that a sequence $\left(y_{s}\right)$ is $i$-flat with respect to the sequence $\left(x_{s}\right)$ if $\left|y_{s}\right| \ll\left|x_{s}\right|^{i}$. We will say that the sequence $\left(y_{s}\right)$ is $i^{-}$-flat with respect to $\left(x_{s}\right)$ if $\left|y_{s}\right| \leq C\left|x_{s}\right|^{i}$ for a constant $C$.

Let

$$
\mathcal{G}(\mathcal{S})=\bigcup_{i \leq p}\left\{\left(x ; T_{x} X_{i}\right) \in X \times \mathbb{G}^{d} \mid x \in X_{i}\right\} .
$$

For $T \in \mathbb{G}^{d}$ let $\Sigma(T)=\left\{v \in \operatorname{Lin}(N ; Y) \mid \Gamma_{v} \not h T\right\}$.

Definition 2.1.2. Let $i, j \in \mathbb{R} \cup \mathbb{R}^{-}$. We will say that $(v ; \mathcal{S})$ is $\left(t^{i, j}\right)$ if no sequence $\left(x_{s} ; y_{s} ; T_{s}\right)$ of points of $\mathcal{G}$ tending to 0 satisfies: $\left|y_{s}-v\left(x_{s}\right)\right|$ is $i$-flat and $d\left(y-d_{x_{s}} v ; \Sigma\left(T_{s}\right)\right)$ is $j$-flat.

For $i \in \mathbb{R}$, we will say that $(v ; \mathcal{G})$ is $\left(t^{i}\right)$ if it is $\left(t^{i, i-1}\right)$.

REMARK 1. Condition $\left(t^{i}\right)$ can be characterized by transversality to all the strata $X_{s}$ of $C^{i}$ direct transversals to $Y$ (see [9]). 
The $\left(t^{i}\right)$ conditions will provide our criteria of finite determinacy. To be able to express these criteria analytically we are going to give some explicit characterizations of the $\left(t^{i}\right)$ conditions.

REMARK 2. For $i \geq 1$ (in $\mathbb{R}$ ) the following explicit characterization of $\left(t^{i}\right)$ will provide us an analytic condition for determinacy. Define the horn neighborhood of $v$ of order $i$ to be the following set:

$$
H(v ; i ; C)=\left\{q=(x ; t) \in \mathbb{R}^{n} \times \mathbb{R}^{m}|| v(x)-\left.t|\leq C| x\right|^{i}\right\} .
$$

Let

$$
\mu(L ; T)=\inf _{|u|=1, u \in T}\|L u\|_{2}
$$

whenever $L: \mathbb{R}^{d} \rightarrow Y$ is a linear map and $T \subseteq \mathbb{R}^{d}$ a vector space (here $\|\cdot\|_{2}$ is the euclidian norm). Then for $i \geq 1$ we get the following characterization of condition $\left(t^{i}\right)$. The couple $(v ; \mathcal{S})$ is $\left(t^{i}\right)$ if and only if there exists a horn neighborhood of $v$ of order $i$ such that for any $x$ in this set,

$$
\mu\left(d_{(x ; y)}(y-v(x)) ; T_{(x ; y)} X_{k}\right) \geq \varepsilon|x|^{i-1}
$$

where $X_{k}$ is the stratum containing the point $x$ and $\varepsilon>0$.

For $i$ negative we may relate condition $\left(t^{i}\right)$ to the Kuo-Verdier condition. We define

$$
\tau(U ; V)=\sup \frac{\left|\pi_{V}^{\perp}(u)\right|}{\left|\pi_{V}(u)\right|} .
$$

Then in [9] the following is proved:

Lemma 2.1.3. For all $T \in \mathbb{G}^{d}, \tau(Y ; T)=1 / d(0 ; \Sigma(T))$.

We say that the stratification $\mathcal{S}$ is strongly Verdier regular with exponent $i$ (at the origin) with respect to $Y$ if there exists a neighborhood of the origin such that for each stratum $X_{k}$,

$$
\tau\left(Y ; T_{x} X_{k}\right) \ll|x-\pi(x)|^{i} .
$$

By the above lemma we see that condition $\left(t^{0^{-}, i^{-}}\right)$is actually the strong Verdier condition with exponent $1-i$ at any point of $Y$.

Note that by definition of $\tau$ the strong Verdier condition with exponent $j$ implies (by standard arguments, see [6], [7], [12]) that we are able to construct a vector field tangent to the strata, extending a given vector field on $Y$ and satisfying

$$
|w(q)-w(\pi(q))| \leq C(q)|q-\pi(q)|^{j}
$$

in a sufficiently small neighborhood of $Y$ with $C$ a continuous function tending to zero when $q$ approaches $Y$. 
2.2. Deformation of transversals. We define the notion of deformation of transversals as in [9].

Let $\varrho \in \mathbb{R}$ and $m^{\prime} \in \mathbb{N}$. Let $U$ be a neighborhood of the origin in $\mathbb{R}^{m^{\prime}}$. Let $g, h_{1}, \ldots, h_{m^{\prime}}: N \rightarrow Y$ be smooth functions on $U$ satisfying $\left|h_{r}(x)\right|=o\left(|x|^{\varrho}\right)$ (resp. $\left.\left|h_{r}(x)\right|=O\left(|x|^{\varrho}\right)\right)$ and $\left|d_{x} h_{r}\right|=o\left(|x|^{\varrho-1}\right)\left(\right.$ resp. $\left.\left|d_{x} h_{r}\right|=O\left(|x|^{\varrho}\right)\right)$ for any $r$. Then

$$
\begin{gathered}
F: N \times U \rightarrow N \times Y, \\
F(x ; u)=(x, f(x, u))=\left(x, f_{u}(x)\right)=\left(x, g(x)+\sum_{r=1}^{m^{\prime}} u_{r} h_{r}(x)\right),
\end{gathered}
$$

is called a deformation of contact $\varrho$ (resp. of contact $\varrho^{-}$).

Deformations of transversals induce a pull-back transformation over the graphs of maps from $N \times Y$ into $\mathbb{G}^{d}$. We define it as in [9].

If for each point $p \in U$ the mapping $F$ is transverse to the strata we may define $F^{*} \mathcal{S}$ as the stratification of $F^{-1}(X)$ given by the manifolds $F^{-1}\left(X_{k}\right)$.

Then let us define the push-forward of a transversal. Let $u$ be a transversal to $U$. We will denote by $F_{*} u$ the transversal defined by $v(x)=g(x)+$ $\sum_{r=1}^{m^{\prime}} u_{r}(x) h_{r}(x)$. The map $F$ then sends the graph of $u$ onto the graph of $v$.

Extend also addition to $\mathbb{R} \cup \mathbb{R}^{-}$by setting $a^{-}+b=a+b^{-}=a+b$ and $a^{-}+b^{-}=(a+b)^{-}$.

TheOREM 2.2.1 (see [9]). Let $F$ be a deformation of contact $\alpha$. If the couple $\left(F^{*} u ; \mathcal{G}\right)$ is $\left(t^{\alpha+\varrho}\right)$ then $\left(u ; F_{*} \mathcal{G}\right)$ is $\left(t^{\varrho}\right)$.

Let $i \in \mathbb{R} \cup \mathbb{R}^{-}$and $a \in \mathbb{R}$. In particular the following corollary will be interesting for us:

COROLlary 2.2.2. Let $F$ be a deformation of contact $i+a$ and $u=F^{*} 0$. If $\left(F^{*} u ; \mathcal{G}\right)$ is $\left(t^{i}\right)$ then $\left(0 ; F_{*} \mathcal{G}\right)$ is strongly Verdier regular with exponent $a+1$.

3. On the volume of stratified families. For $r \in \mathbb{R}$, strictly positive, we denote by $B(0 ; r)$ the ball of center 0 and radius $r$ and by $S(0 ; r)$ the sphere of center 0 and radius $r$.

Let $A$ be a subanalytic subset of $\mathbb{R}^{n} \times \mathbb{R}^{m}$. We will consider such a subset as a family of subanalytic subsets of $\mathbb{R}^{n}$ parametrized by $\mathbb{R}^{m}$. For $U \subseteq \mathbb{R}^{m}$ we denote by $A_{U}$ the subfamily $\left\{q=(x ; t) \in \mathbb{R}^{n} \times \mathbb{R}^{m} \mid q \in A, t \in U\right\}$, and for $t \in \mathbb{R}^{m}$ we denote by $A_{t}$ the fiber of $A$ at $t$, that is, $\left\{x \in \mathbb{R}^{n} \mid q=(x ; t) \in A\right\}$.

We denote by $\mathcal{H}^{l}$ the $l$-dimensional Hausdorff measure. We are going to study the Hausdorff measure of subanalytic sets. For this if $X$ is the germ of a subanalytic subset of $\mathbb{R}^{n}$ at 0 we define the functions $\psi(X ; r)$ and $\widetilde{\psi}$ in the following way:

$$
\psi(X ; r)=\mathcal{H}^{l}(X \cap B(0 ; r))
$$


where $l$ is the Hausdorff dimension of $X$, and

$$
\widetilde{\psi}(X ; r)=\frac{\psi(X ; r)}{\mu_{l} r^{l}},
$$

where $\mu_{l}$ is the volume of the unit ball in $\mathbb{R}^{l}$. In [5] J.-M. Lion and J.-P. Rolin have proved that the function $\psi$ has an expansion in $r$ and $\ln r$. This is also proved in [1] and [8].

Theorem 3.0.3 ([1], [5], [8]). Let $(X ; 0)$ be a subanalytic germ. The function $\psi(X ; r)$ has an expansion

$$
\psi(X ; r)=\sum_{(\alpha ; \beta) \in \mathbb{N}^{2}, \alpha \geq p l} a_{\alpha, \beta} r^{\alpha / p} \ln ^{\beta} r .
$$

The expansion of $\psi$ up to order $k$ will designate the terms having an exponent in $r$ less than or equal to $k$.

Note that the limit

$$
\theta(X ; x):=\lim _{r \rightarrow 0} \frac{\mathcal{H}^{l}(X \cap B(x ; r))}{\mu_{l} r^{l}}
$$

is the density of $X$ (or the Lelong number). This number was originally introduced for complex analytic sets and then generalized to real subanalytic sets by K. Kurdyka and G. Raby in [4]. It gives interesting information on the behavior of the volume of a germ. In [11] the author has studied the variation of the density along stratified spaces.

We recall some results which we will need in the next section and which can be found in [11].

3.1. Volumes, multiplicities and isotopies. In this section we bound the variation of the volume through the isotopies which are $i$-approximations of the identity.

Definition 3.1.1. Let $A$ and $B$ be two subanalytic families of sets and $i$ be a positive real number. We define an $i$-approximation of the identity to be a family of mappings which induces a family of germs of homeomorphisms $h:(A ; 0) \rightarrow(B ; 0)$ of type $h(x ; t)=\left(h_{t}(x) ; t\right)$ such that for every $t$,

$$
\left|h_{t}(x)-x\right| \ll|x|^{i}
$$

for all $x \in B(0 ; r)$ with $r$ sufficiently close to zero.

REMARK 3. Note that if $d_{\mathcal{H}}$ denotes the Hausdorff distance between compact sets, that is,

$$
d_{\mathcal{H}}(A ; B)=\max \left(\sup _{x \in A} d(x ; B) ; \sup _{x \in B} d(x ; A)\right)
$$

then it follows from the definition of $i$-approximation of the identity that

$$
d_{\mathcal{H}}\left(A_{t} \cap B(0 ; r) ; B_{t} \cap B(0 ; r)\right) \ll r^{i},
$$

with the notations of the definition above. 
In [11] the following proposition is proved (in a slightly more general setting; the following one is the case $\alpha \ll r^{i}$ in [11]).

Proposition 3.1.2. Let $i$ be a strictly positive real number. Let $h: A \rightarrow$ $B$ be an $i$-approximation of the identity and let $P$ be in $\mathbb{G}(n ; l)$ (where $l=$ $\operatorname{dim} A_{t}=\operatorname{dim} B_{t}$ for any $\left.t \in \mathbb{R}^{m}\right)$. Then for any compact subset $V$ of $\mathbb{R}^{m}$ there exists a constant $C$ and a subanalytic subset $K(P ; r ; t) \subseteq P$ satisfying

$$
\psi(P \cap B(0 ; r) \backslash K(P ; r ; t) ; r) \ll r^{i+l-1},
$$

and such that for any $x \in K(P ; r ; t)$ and $t \in V$,

$$
\operatorname{card}\left(\pi_{P}^{-1}(x) \cap A_{t} \cap B(0 ; r)\right)=\operatorname{card}\left(\pi_{P}^{-1}(x) \cap B_{t} \cap B(0 ; r)\right) .
$$

This proposition has been proved to study the behavior of the density of stratified sets satisfying the Whitney condition. Actually using the CauchyCrofton formula it allows us to bound the variation of the volume. It is the key point in the proof of the theorem below (again the reader is referred to [11] for the proof).

THEOREM 3.1.3. Let $i \in \mathbb{R}$ and $h: A \rightarrow B$ be an $i$-approximation of the identity. Let $V$ be a compact subanalytic subset of $\mathbb{R}^{m}$. Then there exists a constant $C$ such that for all $t$ in $V$,

$$
\left|\psi\left(A_{t} ; r\right)-\psi\left(B_{t} ; r\right)\right| \ll r^{i+l-1} \text {. }
$$

Let us give another definition we shall need in the next section. Let $P$ be an $l$-dimensional vector space of $\mathbb{R}^{n}$ and $i \in \mathbb{R}$ with $i \geq 1$. For $j \in \mathbb{N}$ we set

$$
K_{j}^{P}(A \cap B(0 ; r))=\left\{x \in P \mid \operatorname{card}\left(\pi_{P}^{-1}(x) \cap A_{t} \cap B(0 ; r)\right)=j\right\} .
$$

Definition 3.1.4. We say that $j$ is a multiplicity of $A$ for $P$ of order $i$ if $0 \in \operatorname{cl}\left(K_{j}^{P}(A)\right)$ and if there exists a positive constant $C$ such that for any $r$ sufficiently close to zero,

$$
\widetilde{\psi}\left(K_{j}^{P}(A \cap B(0 ; r)) ; r\right) \geq C r^{i} .
$$

We will denote by $m(P ; A ; i)$ the set of multiplicities of order $i$ of $A$ for $P$.

Another consequence of Proposition 3.1.2 is the invariance of the set $m(P ; A ; i)$ under $i$-approximation of the identity for any $P$.

3.2. Rugose isotopies. In this section we are going to prove an isotopy lemma which will be used to prove finite determinacy in the next section.

Given a subset $A$ of $\mathbb{R}^{n}$ and $\varepsilon>0$, we denote by $A^{\varepsilon}$ the set of points $q \in \mathbb{R}^{n}$ such that $d(q ; A) \leq \varepsilon$.

Proposition 3.2.1. Let $A$ be a subanalytic subset of $\mathbb{R}^{n}$ of dimension $l$. Let $B \subseteq A$ be a subanalytic subset of dimension $k<l$. Then there exists a constant $C$ such that for all $\varepsilon \in] 0 ; 1]$ and for all real $r$ small enough,

$$
\psi\left(B^{\varepsilon} \cap A ; r\right) \leq C r^{l-1} \varepsilon .
$$


Proof. The jacobian of the distance function to a subset of $\mathbb{R}^{n}$ is equal to 1 wherever this function is differentiable. Moreover the family $B^{\prime}=\{(x ; \alpha) \in$ $A \times \mathbb{R} \mid d(x ; B)=\alpha\}$ is a subanalytic family. So using Proposition 3.0.4 of [11] we deduce that there exists a constant $C$ such that for all $\alpha \in] 0 ; 1]$ we have $\psi\left(B_{\alpha}^{\prime} ; r\right) \leq C r^{l-1}$. So, we can write

$$
\psi\left(B^{\varepsilon} \cap A ; r\right)=\int_{B^{\varepsilon} \cap A \cap B(0 ; r)} d \mathcal{H}^{l} \leq \int_{\alpha \in] 0 ; \varepsilon[} \psi\left(B_{\alpha}^{\prime} ; r\right) d \mathcal{H}^{1} \leq C r^{l-1} \varepsilon .
$$

Now let $X \subseteq \mathbb{R}^{n} \times \mathbb{R}^{m}$ be a subanalytic family of sets of dimension $l$. Assume, as in Section 2, that $X$ has a stratification $\mathcal{S}=\left\{Y, X_{1}, \ldots, X_{p}\right\}$. We have:

THEOREM 3.2.2. If $\mathcal{S}$ satisfies the strong Verdier condition with exponent $i \geq 1$ then there exist a neighborhood $U$ of the origin and a trivialization

$$
h: \pi^{-1}(U) \backslash B \rightarrow N \times U \backslash B^{\prime},
$$

where $B\left(\right.$ resp. $\left.B^{\prime}\right)$ is a subanalytic subfamily of $X\left(\right.$ resp. of $\left.X_{0} \times U\right)$ such that $\psi\left(B_{t} ; r\right) \ll r^{i+l-1}$ (resp. $\left.\psi\left(B_{t}^{\prime} ; r\right) \ll C r^{i+l-1}\right)$ for $t$ in $U$, which is an $i$-approximation of the identity preserving the $X_{j} \cap \pi^{-1}(U)$.

Proof. We prove this result by a similar method to that used in [11]. Let $A^{k}$ denote the $k$-skeleton of the stratification $\mathcal{S}$, that is, the union of the strata of dimension less than or equal to $k$. We define the isotopy (and the families $B$ and $B^{\prime}$ ) on $A^{k}$ inductively on $k$. The result is obvious for $k=\operatorname{dim} Y$. Assume that it is true at a rank $k$. Then by Remark 3 we have

$$
d_{\mathcal{H}}\left(\pi^{-1}(t) \cap A^{k} \cap B(t ; r) ; \pi^{-1}\left(t^{\prime}\right) \cap A^{k} \cap B\left(t^{\prime} ; r\right)\right) \ll r^{i}
$$

for $t$ and $t^{\prime}$ fixed in $Y$. The isotopy is constructed by lifting a basis of $Y$ to a stratified vector field tangent to strata and rugose with exponent $i$ (see [6] or [12]). As the construction can be carried out with all vectors of a basis of $Y$ successively we will assume that $\operatorname{dim} Y=1$.

Let $w$ be a constant unit vector field on $Y$. By (2.2) we may extend $w$ to a vector field tangent to the strata and satisfying (2.2). Let $\phi$ be the oneparameter group generated by this vector field. Write $\phi=\left(\phi_{1} ; \phi_{2}\right) \in N \times Y$; then by Gronwall's Lemma we have

$$
d(q ; Y) e^{-M s} \leq d(\phi(q ; s) ; Y) \leq e^{M s} d(q ; Y)
$$

for a constant $M$. Hence the ratio $d(q ; Y) / d\left(\phi_{r}(q ; s) ; Y\right)$ is bounded below and above. This proves that the integral curves cannot join $Y$ or leave a neighborhood of $Y$ in a finite time. Moreover by the mean value theorem,

$$
\left|\phi_{1}(q ; t)-q\right| \leq t \sup _{s \in[0 ; t]}\left|\pi^{\perp}(w(\phi(q ; s)))\right| \leq C(q) \sup _{s \in[0 ; t]} d(\phi(q ; s) ; Y)^{i} .
$$

Hence as the ratio $d(q ; Y) / d\left(\phi_{r}(q ; s) ; Y\right)$ is bounded we get

$$
\left|\phi_{1}(q ; t)-q\right| \leq C(q) d(q ; Y)^{i}
$$


(with maybe a different function $C$ tending to zero when we approach $Y$ ). This proves that the mapping will define an $i$-approximation of the identity.

Therefore to complete the induction step it suffices to prove existence of integral curves starting from a point $q \in A^{k+1}$ and sufficiently far from $A^{k}$. More precisely, let

$$
B=\left\{q \in A^{k+1} \mid d\left(q ; A^{k}\right) \leq \sqrt{C(q)} d(q ; Y)^{i}\right\} .
$$

Now by (3.5) and (3.6) we see that if $q \in A^{k+1}$ does not belong to $B$ then $\phi(q ; s)$ cannot fall in $A^{k}$. This establishes the existence of the desired integral curves. Moreover, if we set

$$
h: A^{k+1} \backslash B \rightarrow \pi^{-1}(0) \times U, \quad q=(x ; t) \mapsto\left(\phi_{1}(q ;-t) ; t\right),
$$

then $h$ is a homeomorphism onto its image for each $t$ and an $i$-approximation of the identity. Let

$$
B^{\prime}=\left(A_{0} \times U\right) \backslash h(A \backslash B) .
$$

Note that again as a consequence of (3.5) and (3.6) we have

$$
B^{\prime} \subseteq\left\{q \in A^{k+1} \mid d\left(q ; A^{k}\right) \leq C(q) d(q ; Y)^{i}\right\}
$$

(sufficiently close to $Y$ ). Therefore to finish the proof we just remark that (3.7) (resp. (3.8)) and Proposition 3.2.1 show that at the last step of the induction we have $\psi\left(B_{t} ; r\right) \ll r^{i+l-1}$ (resp. $\psi\left(B_{t}^{\prime} ; r\right) \ll r^{i+l-1}$ ) as required.

4. Finite determinacy of the volume and multiplicities. In this section for $a \in \mathbb{R}$ we denote by $[a]$ the greatest integer less than or equal to $a$.

4.1. Transversal sections of a stratified set. Here we will give some determinacy theorems for the volume of a transversal section to a stratified set. More precisely, we are going to give explicit sufficient conditions for the expansion of $\psi\left(\Gamma_{v} \cap X ; r\right)$ to be determined by that of $v$ up to a certain order. The results on the determination of the volume of the zero locus of a given map will be given in the next section and will be deduced from those of this section since the intersection of the graph with the source axis is precisely the zero locus.

For this section we fix a stratified space $(X ; \mathcal{S})$ where $\mathcal{S}=\left\{Y ; X_{1}, \ldots, X_{p}\right\}$. We assume that $X$ is subanalytic. We recall that $Y=\mathbb{R}^{m}, N=\mathbb{R}^{n}$ and $X \subseteq N \times Y=\mathbb{R}^{d}$.

TheOREM 4.1.1. Let $v: N \rightarrow Y$ be a subanalytic transversal and let a and $j$ be positive real numbers with $j \geq 1$. If $(v ; \mathcal{S})$ satisfies the $\left(t^{j}\right)$ condition then the expansion to order a of $\widetilde{\psi}\left(\Gamma_{v} \cap X ; r\right)$ only depends on the $[j+a]$-jet of $v$ at 0 . Moreover, for every $P \in \mathbb{G}(d ; l)$ with $l=\operatorname{dim} X-m, m\left(P ; \Gamma_{v} \cap X ; a\right)$ only depends on the $[j+a]$-jet of $v$. 
Proof. Let $u$ and $v$ be two $C^{[j+a]}$ mappings having the same $[j+a]$-jet. Let $F(x ; t)=(x ; v(x)+t h(x))$ where $h(x)=u(x)-v(x)$. Then $F$ is a deformation of contact $[j+a]$ and $F_{*} 0=v$.

Thanks to Corollary 2.2.2 we know that $\left(0 ; F^{*} \mathcal{S}\right)$ is strongly Verdier regular with exponent $a+1$ at any point of $\{0\} \times \mathbb{R}$. So by Theorem 3.2.2 there exists an isotopy $H: \mathbb{R}^{d^{\prime}} \times[0 ; 1] \rightarrow \mathbb{R}^{d^{\prime}}$ (where $d^{\prime}=n+1$ ) preserving the strata $F^{-1}\left(X_{k}\right)$ which is an $(a+1)$-approximation of the identity. For any map $u: N \rightarrow Y$ let $\phi_{u}(x)=(x ; u(x))$.

Then we set

$$
\widetilde{H}: \Gamma_{v+h} \rightarrow \Gamma_{v}, \quad q \mapsto \widetilde{H}(q)=\phi_{v} \circ H_{1} \circ \phi_{-v-h}(q),
$$

where $H_{1}(x)=H(x ; 1)$. As a consequence we have

$$
\begin{aligned}
|\widetilde{H}(q)-q| & =\mid \phi_{v}\left(H_{1}\left(\phi_{-v-h}(q)\right)-q \mid\right. \\
& =\left|\phi_{v}\left(H_{1}\left(\phi_{-v-h}(q)\right)\right)-\phi_{v}\left(\phi_{-v}(q)\right)\right| \\
& \lesssim\left|H_{1}\left(\phi_{-v-h}(q)\right)-\phi_{-v}(q)\right| \quad \text { for } \phi_{v} \text { is Lipschitz } \\
& \lesssim\left|H_{1}\left(\phi_{-v-h}(q)\right)-\phi_{-v-h}(q)\right|+\left|\phi_{-v-h}(q)-\phi_{-v}(q)\right| \\
& \ll d(q ; Y)^{a+1} \quad \text { since } j^{[j+a]} h(0)=0 .
\end{aligned}
$$

Therefore the mapping $\widetilde{H}$ is an $(a+1)$-approximation of the identity. But by Theorem 3.1.3 this implies that the expansions of the volume of $\Gamma_{v} \cap X$ and $\Gamma_{u} \cap X$ coincide. Moreover by Theorem 3.1.2 the multiplicities also coincide up to order $a$.

REMARK 4. Thus by Remark 2 we may make explicit the criterion of the above theorem. More precisely, inequality (2.1) provides an analytic criterion for the determinacy of the volume and multiplicities of transverse sections.

Note also that by the Łojasiewicz inequality the above theorem yields finite determinacy for every analytic mapping with an isolated singularity.

In particular in the case of a function we get the following interesting corollary:

COROLlary 4.1.2. If $v$ is a subanalytic function with an isolated singularity at the origin satisfying $\left|\partial_{x} v\right| \geq C|x|^{j-1}$ in a horn neighborhood, then the expansion of $\widetilde{\psi}\left(\Gamma_{v} \cap X ; r\right)$ up to order $a$ is determined by its $[j+a]$-jet. In particular the density $\theta\left(\Gamma_{v} \cap X\right)$ just depends on the $[j]$-jet (this is the case of $a=0)$.

In the case where $j<1$, Theorem 4.1.1 is no longer true. The problem comes indeed from $h$ which does not decrease sufficiently fast. Nevertheless we have the following result which will be useful for the next section.

Let $v$ be a direct transversal and $A$ a vector space directly transverse to $Y$. Let $\pi_{A}$ be the projection onto $A$ along $Y$. Then $\pi_{A}$ induces a diffeomorphism of $\Gamma_{v}$ onto $A$. 
Proposition 4.1.3. Assume that $X$ is a subanalytic set. Let $A \in \mathbb{G}(d ; n)$ be transverse to $Y, a, j \in \mathbb{R}$, and $v$ a subanalytic direct transversal. If $(v ; \mathcal{S})$ satisfies condition $\left(t^{j}\right)$ then the expansion up to order a of $\widetilde{\psi}\left(\pi_{A}\left(X \cap \Gamma_{v}\right)\right)$ is determined by the $[j+a]$-jet of $v$. Moreover, the multiplicities of $\pi_{A}\left(X \cap \Gamma_{v} ; r\right)$ of order less than or equal to a are determined by the $[j+a]$-jet of $v$.

Proof. Here we set again $F(q)=v(x)+t h(x)$ where $q=(x ; t)$ and $h$ is a function having a zero $[j+a]$-jet.

Actually we have $F^{-1}(X)=\left\{(x ; t) \in \mathbb{R}^{n} \times \mathbb{R} \mid\left(x ; F_{t}(x)\right) \in X\right\}$. We recall that $\pi^{\perp}$ denotes the orthogonal projection on the orthogonal of $Y$.

Hence

$$
F^{-1}(X) \cap(N \times\{t\})=\pi^{\perp}\left(X \cap \Gamma_{F_{t}}\right) \times\{t\} .
$$

So

$$
\begin{aligned}
& F^{-1}(X) \cap N \times\{0\}=\pi^{\perp}\left(X \cap \Gamma_{v}\right) \times\{0\}, \\
& F^{-1}(X) \cap N \times\{1\}=\pi^{\perp}\left(X \cap \Gamma_{u}\right) \times\{1\} .
\end{aligned}
$$

We set

$$
h=\left(\pi_{\mid A}^{\perp}\right)^{-1} \circ H_{1} \circ \pi^{\perp}
$$

where $H_{1}$ is constructed as in the proof of Theorem 4.1.1. So $h$ is an approximation of the identity to the same order as $H_{1}$, which proves the result.

In the case where $j=1^{-}$we also obtain an interesting result:

Proposition 4.1.4. Let $A$ and $B$ be vector spaces directly transverse to $Y$ to 0 . If all the $\left(Y ; X_{j}\right)$ satisfy condition (a) of Whitney then

$$
\theta\left(\pi_{A}(B \cap X) ; 0\right)=\theta(A \cap X ; 0) .
$$

Moreover, for every $P \in \mathbb{G}(n ; l)$, the set $\left.m\left(\pi^{\perp}(B \cap X)\right) ; 1 ; P\right)$ does not depend on $B$.

Proof. The (a) condition of Whitney actually implies $\left(t^{1^{-}}\right)$for any direct transversal $v$ (see Lemma 2.1.3). The proof is very similar to that of Theorem 4.1.3. The only difference is that $a=1^{-}$and $j=0^{-}$and the deformation will be of contact $1^{-}$(recall that the density is the constant term of $\widetilde{\psi}$ ).

REMARK 5. In the situation of the above proposition it is not true that the number $\theta(B \cap X ; 0)$ is independent of $B$.

4.2. $S V$-sufficiency of jets. In this section we consider a stratification $\mathcal{S}=\left\{\{0\}, X_{1}, \ldots, X_{s}\right\}$ of $N$. Let

$$
\mathcal{S}_{0}=\left\{Y, X_{1} \times\{0\}, \ldots, X_{s} \times\{0\}\right\} .
$$

Hence the zero locus of a mapping $v$ is the intersection of the graph of $v$ with $N \times\{0\}$.

From Theorem 4.1.3 we may deduce explicit criteria for determination of the volume and multiplicities. 
TheOrem 4.2.1. Suppose $X$ is a subanalytic set and $v$ a subanalytic function. If $\left(v ; \mathcal{S}_{0}\right)$ is $\left(t^{j}\right)$ then the expansion up to order a of $\widetilde{\psi}\left(X \cap v^{-1}(0) ; r\right)$ is determined by $z=j^{[j+a]} v(0)$. Moreover, in this case, for each vector subspace $P$, the multiplicities of $X \cap v^{-1}(0)$ for $P$ are determined by $z$ up to order $a$.

Proof. This is actually a consequence of Proposition 4.1.3. More precisely it is the case where $A=N \times\{0\}$; then the mapping $\pi_{A_{\mid X}}$ is just the identity.

REMARK 6. As in the above section we get an analytic criterion for determinacy via inequality $(2.1)$. We know that condition $\left(t^{i}\right)$ is satisfied by $\left(v ; \mathcal{S}_{0}\right)$ if there exists a horn neighborhood of type

$$
\left\{\left.x \in \mathbb{R}^{n}|| v(x)|\leq C| x\right|^{i}\right\}
$$

on which we have a constant $C$ such that

$$
\left|d_{x} v(w)\right| \geq C|x|^{i-1}
$$

for any unit vector $w \in \mathbb{R}^{n}$.

As a consequence we get the following result:

THEOREM 4.2.2. Let $v$ be an analytic function with an isolated singularity at the origin such that

$$
\left|\partial_{x} v\right| \geq C|x|^{j-1}
$$

in a neighborhood as in the above remark. Then the terms of the expansion of $\widetilde{\psi}\left(v^{-1}(0) ; r\right)$ up to order a are determined by its $[j+a]$-jet. In particular in this case the number $\theta\left(v^{-1}(0) ; 0\right)$ only depends on the $[j]$-jet of the function $v$.

Let us point out that by the Łojasiewicz inequality we may find an order of determination for every function with an isolated singularity at the origin.

\section{References}

[1] G. Comte, J.-M. Lion et J.-P. Rolin, Nature log-analytique du volume des sousanalytiques, Illinois J. Math. 44 (2000), 884-888.

[2] S. Koike, $C^{0}$-sufficiency of jets via blowing-up, J. Math. Kyoto Univ. 28 (1988), 605-614.

[3] T. C. Kuo, Characterizations of v-sufficiency of jets, Topology 11 (1972), 115-131.

[4] K. Kurdyka et G. Raby, Densité des ensembles sous-analytiques, Ann. Inst. Fourier (Grenoble) 39 (1989), 753-771.

[5] J.-M. Lion et J.-P. Rolin, Intégration des fonctions sous-analytiques et volumes des sous-ensembles sous-analytiques, ibid. 48 (1998), 755-767.

[6] J. Mather, Notes on topological stability, Harvard Univ. 1970.

[7] P. Orro et D. J. A. Trotman, Cône normal et régularités de Kuo-Verdier, Bull. Soc. Math. France 130 (2002), 71-85. 
[8] A. Parusiński, On the preparation theorem for subanalytic functions, in: New Developments in Singularity Theory (Cambridge, 2000), NATO Sci. Ser. II Math. Phys. Chem. 21, Kluwer, Dordrecht, 2001, 193-215.

[9] D. J. A. Trotman and L. C. Wilson, Stratifications and finite determinacy, Proc. London Math. Soc. (3) 78 (1999), 334-368.

[10] G. Valette, Détermination et stabilité du type métrique des singularités, thèse de doctorat.

[11] -, Volume, density and equisingularity conditions, preprint.

[12] J.-L. Verdier, Stratifications de Whitney et théorème de Bertini-Sard, Invent. Math. 36 (1976), 295-312.

[13] C. T. C. Wall, Finite determinacy of smooth map-germs, Bull. London Math. Soc. 13 (1981), 481-539.

Institute of Mathematics

Jagiellonian University

Reymonta 4

30-059 Kraków, Poland

E-mail: Guillaume.Valette@im.uj.edu.pl

Reçu par la Rédaction le 29.11.2004

Révisé le 9.3.2005 